\title{
Estudo da incorporação de material laterítico ferroaluminoso na taxa de queima de compósito de matriz termofixa
}

\author{
Study of the incorporation of iron-aluminous lateritic \\ material in the burning rate of thermoset \\ matrix composites
}

\begin{abstract}
Bruno Henrique Alves Mendes ${ }^{1}$, Sylvester Stallone Pontes Sales ${ }^{1}$, Lesley Glenda Sales dos Santos ${ }^{1}$, Edinaldo José de Sousa Cunha ${ }^{3}$, João Nazareno Nonato Quaresma ${ }^{1,2}$, José Antônio da Silva Souza ${ }^{1,2}$
\end{abstract}

\footnotetext{
${ }^{1}$ Universidade Federal do Pará, Programa de Pós-Graduação em Engenharia Química - (PPGEQ/ITEC/UFPA). Rua Augusto Corrêa, 01, CEP: 66075-110, Pará, Belém, Brasil.

${ }^{2}$ Universidade Federal do Pará, Programa de Pós-Graduação em Engenharia de Recursos Naturais da Amazônia (PRODERNA/ITEC/UFPA). Rua Augusto Corrêa, 01, CEP: 66075-110, Pará, Belém, Brasil.

${ }^{3}$ Universidade Federal do Pará, Faculdade de Engenharia de Materiais (FEMAT/CAMPANIN/UFPA). Cidade Nova IV, WE 26, CEP: 67113-901, Pará, Ananindeua, Brasil

e-mail: bhmendes15@gmail.com, sylvestersales@hotmail.com, lesleyglenda@gmail.com, quaresma@ufpa.br, jass@ufpa.br, cunha.edinaldo@gmail.com;
}

\section{RESUMO}

Os compósitos são versáteis e podem ser aplicados em diversos setores dependendo das necessidades exigidas e de suas características, de modo a conciliar fatores como segurança, meio ambiente e economia. Os materiais lateríticos estão presentes em grande parte do território nacional e podem apresentar teores elevados de óxi-hidróxidos de ferro e alumínio, podendo influenciar significativamente nas propriedades térmicas de compósitos poliméricos. O material utilizado no trabalho é originário da Serra do Piriá, localizada no município de Vizeu, Estado do Pará. O trabalho consistiu na caracterização deste material e produção de compósitos utilizando o mesmo como carga, em uma matriz de poliéster isoftálico, os quais foram testados através de ensaios de flamabilidade. Foram realizadas análises de granulometria a laser, espectroscopia de fluorescência de raios-X (FRX), infravermelho por transformada de Fourier (FTIR), termogravimetria (TG) e calorimetria exploratória diferencial (DSC). Os compósitos foram fabricados segundo processo hand lay-up em molde fechado, com compressão de 2,7 toneladas por 20 minutos. As frações volumétricas utilizadas para a fabricação dos compósitos foram de 10, 20 e $30 \%$ e os ensaios de flamabilidade foram conduzidos segundo a norma ASTM D 635-03. Como resultados, o material apresentou diâmetro médio igual a 23,5 $\mu \mathrm{m}$, constituído por $61,13 \%$ de $\mathrm{Fe}_{2} \mathrm{O}_{3}$ e $18,63 \%$ de $\mathrm{Al}_{2} \mathrm{O}_{3}$, ratificando a presença destes óxidos, bandas características a $3448 \mathrm{~cm}^{-}$ ${ }^{1}$ indicam a presença de cátions trivalentes de $\mathrm{Al}^{3+}$ ou $\mathrm{Fe}^{3+}$. Quanto ao comportamento calorimétrico, o mesmo apresentou transformações de fases em uma faixa de temperatura adequada ao retardo de chamas, com perda de massa significativa a partir de aproximadamente $270{ }^{\circ} \mathrm{C}$. Nos compósitos com $30 \%$ de material laterítico a propagação de chamas reduziu-se em 69,86 \% com relação a matriz de poliéster, o que viabiliza o estudo de sua aplicação em compósitos.

Palavras-chave: Laterita, Ferro, Alumínio, Caracterização.

\section{ABSTRACT}

The composite materials are versatile and can be applied in different sectors depending on the required needs and their characteristics, in order to reconcile factors such as safety, environment, economy. Lateritic materials are present in a large part of the national territory and may exhibit high levels of iron and aluminum oxide and hydroxides, and may have a significant influence on the thermal properties of polymer composites. The material used in the work originated in Piriá ridge, located in the municipality of Vizeu, in the State of Pará. The work consisted of the characterization of this material and the preparation of composites using it as a 
filler in an isophthalic polyester matrix, which were tested through flammability tests. Analyses of laser granulometry, X-Ray fluorescence spectroscopy (XRF) and Fourier Transform Infrared (FTIR), Thermogravimetry (TG) and Differential Scanning Calorimetry (DSC) were performed. The composites were made by hand lay-up process in a closed mold, with compression of 2.7 tons for 20 minutes. The volumetric fractions used to manufacture the composites were 10,20 and $30 \%$ and the flammability tests were conducted according to ASTM D 635-03. As results, the material was composed of fine particles with a mean diameter of 23.5 $\mu \mathrm{m}$, consisting of $61.13 \% \mathrm{Fe}_{2} \mathrm{O}_{3}$ and $18.63 \% \mathrm{Al}_{2} \mathrm{O}_{3}$, confirming the presence of these oxides characteristic bands at $3448 \mathrm{~cm}^{-1}$ indicate the presence of trivalent cations of $\mathrm{Al}^{3+}$ or $\mathrm{Fe}^{3+}$. The calorimetric behavior of this material presented phase transformations in a temperature range suitable for flame retardance, with significant loss of mass from approximately $270{ }^{\circ} \mathrm{C}$. In the composites with $30 \%$ of lateritic material, the flame propagation was reduced by $69.86 \%$ in relation to the polyester matrix, which makes it possible to study its application in composites.

Keywords: Laterite, Iron, Aluminum, Characterization.

\section{INTRODUÇÃO}

A utilização de materiais compósitos é muito antiga e a busca crescente por novas tecnologias põe em foco a necessidade de materiais que atendam as demandas atuais. Nesse contexto, os compósitos poliméricos demostram uma grande flexibilidade de aplicação por apresentar a possibilidade de melhorias em suas propriedades e adequação às exigências de uso. A interação entre as fases matriz e carga é de extrema importância, bem como a melhor dispersão desse material no interior da resina e a escolha do tipo de carga aplicado é condicionado à necessidade de aplicação e às características como rigidez, resistências, peso, rendimento a altas temperaturas, corrosão, dureza e condutividade.

Contudo, os compósitos poliméricos comumente apresentam em sua composição cadeias de hidrocarbonetos que queimam rapidamente e, por esse motivo, necessitam da adição de materiais que atuem no retardo ou extinção da combustão [1]. Essa necessidade estimula o desenvolvimento de pesquisas com o objetivo de disponibilizar novos produtos que contribuam nesse propósito, gerando compósitos com maior resistência térmica, e sendo um dos motivos para a crescente busca por materiais inorgânicos que possam ser incorporados em matrizes poliméricas, como os apresentados nos trabalhos de AL-TAEE et al. [2] e TANG et al. [3] ao estudar o efeito do caulim e do fosfato diamônico nas propriedades térmicas de compósitos, e ZHANG et al. [4], FENG et al. [5], SU et al. [6] e WU et al. [7] ao estudar o efeito da adição de óxidos de $\mathrm{Fe}, \mathrm{Ni}, \mathrm{Mn}$ e Zn, respectivamente.

O retardo de chamas ocorre com a inibição ou interrupção do ciclo do fogo de diferentes maneiras, dentre as quais: utilização de materiais que se decompõem endotermicamente, evitando ou aumentando o tempo necessário para atingir a temperatura de pirólise, como é o caso da alumina tri-hidratada e do hidróxido de magnésio, que liberam vapor d'água gerado pela decomposição de sua estrutura a 200 e $300{ }^{\circ} \mathrm{C}$, respectivamente; utilização de materiais reagentes que geram subprodutos sólidos não inflamáveis a partir de alterações nas cadeias de polímeros degradados, dificultando a transferência de calor; e a utilização de materiais que ao reagir liberem gases não inflamáveis $\left(\mathrm{H}_{2} \mathrm{O}, \mathrm{CO}_{2}, \mathrm{NH}_{3}\right.$, etc.) reduzindo a concentração de oxigênio presente próximo à chama $[1,8]$.

Os materiais lateríticos estão presentes em grande parte do território nacional e seu conceito é aplicado de forma bastante ampla. Segundo COSTA [9] o termo em questão é utilizado para determinar rochas formadas ou em processo de formação através de intemperismo de rochas preexistentes. Esses materiais apresentam teores elevados de óxi-hidróxidos de ferro e alumínio, variando em sua composição o teor dos constituintes e os materiais associados. Podem ser compactos, maciços, coesos e incoesos, com coloração variando entre vermelho, violeta, amarelo, marrom e até branco $[9,10]$.

Com a presença de concentrações consideráveis de óxi-hidróxidos de ferro e alumínio em sua composição, conforme encontrado nos trabalhos de VIEIRA et al. [10], QUAST et al. [11], CASTRO et al. [12] e ZHOU et al. [13], sua aplicação como auxiliar de retardância pode ser promissora, uma vez que esses materiais tendem a se decompor em temperaturas inferiores à $400^{\circ} \mathrm{C}$, liberando vapor d'água em quantidades significativas, podendo atuar na redução da taxa de combustão dos compósitos formados. Nesse sentido, o trabalho objetivou a caracterização química, física e térmica do material laterítico ferroaluminoso identificando suas propriedades e investigando o efeito da adição desses materiais na flamabilidade de compósitos com matriz poliéster insaturado isoftálico. 


\section{MATERIAIS E MÉTODOS}

\subsection{Materiais Utilizados}

O material laterítico ferroaluminoso utilizado no trabalho, oriundo da região da Serra do Piriá, localizada no município de Vizeu, Nordeste do Estado do Pará, foi obtido através do Instituto de Geociências da UFPA (IG/UFPA). A resina poliéster insaturada isoftálica (AM 910), de média reatividade, não acelerada e baixa viscosidade, o catalisador metálico umedecido (Octoato de cobalto - CAT MET 1,5\%) e o iniciador de reticulação (Butanox-M50) foram obtidos junto à AEROJET Brasileira de Fiberglass.

\subsection{Moagem e Classificação}

O material laterítico (Figura 1-a) foi submetido ao processo de moagem em moinho de bolas (CIMAQ S.A. modelo Work Index 005) por 10 minutos. Após esse processo, o material cominuído (Figura 1-b) foi classificado em peneira da série Tyler com abertura de $28 \mathrm{Mesh}(0,6 \mathrm{~mm})$, dando origem a fração utilizada na fabricação dos compósitos (Figura 1-c).

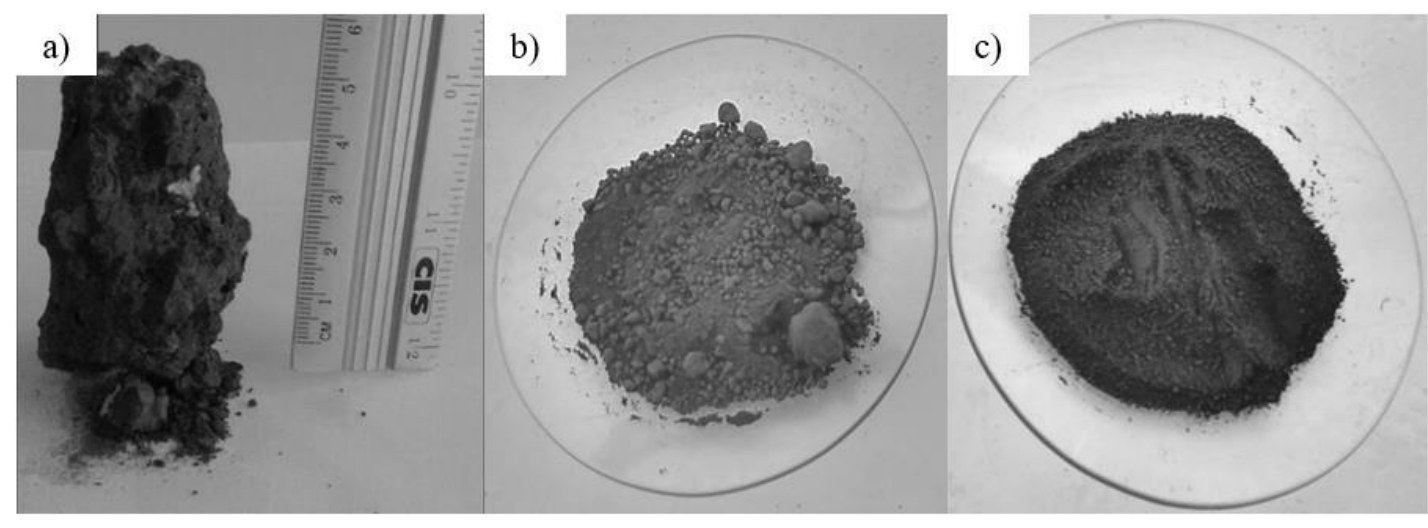

Figura 1: Alterações impostas ao material de partida para adequação à aplicação nos compósitos. a) Laterita Bruta; b) Produto da desagregação em moinho; c) Fração homogeneizada.

\subsection{Granulometria a Laser}

A análise de Granulometria por Difração a Laser foi realizada em um equipamento modelo FRITSCH ANALYSETTE 22 MicroTec Plus. A amostra foi dispersa em água destilada e o equipamento foi operado para varredura entre $0,08-2000 \mu \mathrm{m}, 100$ medições, com obscurecimento de feixe de $14 \%$ e cálculo por Fraunhofer.

\subsection{Espectrometria de Fluorescência de Raios-X (FRX)}

A análise de Espectrometria de Fluorescência de Raios-X foi realizada utilizando o espectrômetro WDS sequencial, modelo Axios Minerals da marca PANalytical, com tubo de raios-X cerâmico, ânodo de ródio (Rh) e máximo nível de potência de 2,4 KW. A amostra foi preparada por disco fundido com $1 \mathrm{~g}$ de amostra e $6 \mathrm{~g}$ de fundente (Tetraborato de Lítio $-\mathrm{Li}_{2} \mathrm{~B}_{4} \mathrm{O}_{7}$ ), fundidos a $1000{ }^{\circ} \mathrm{C}$ por $10 \mathrm{~min}$. A aquisição e tratamento dos dados foi realizada através do software SuperQ Manager da PANalytical.

\subsection{Análise Termogravimétrica (TG) e Calorimetria Exploratória Diferencial (DSC)}

As análises de TG e DSC foram obtidas utilizando-se um equipamento modelo NETZSCH STA 449F3 Júpiter, com analisador térmico simultâneo da NETStanton Redcroft Ltda., forno cilíndrico vertical de platina, faixa de temperatura de $25{ }^{\circ} \mathrm{C}$ até $1000{ }^{\circ} \mathrm{C}$, sob fluxo de nitrogênio $50 \mathrm{~mL} / \mathrm{min}$, razão de aquecimento de 5 ${ }^{\circ} \mathrm{C} / \mathrm{min}$ e cadinho de platina como referência.

\subsection{Processo de Fabricação dos Compósitos}

Inicialmente foram realizados ensaios preliminares para determinação de propriedades importantes para o processo de fabricação dos compósitos, dentre os quais o tempo de ponto de gel e o limite de trabalhabilidade, definindo a fração volumétrica máxima aplicada no trabalho através da fabricação em pequena escala de 
corpos de prova cilíndricos, com diâmetro de $60 \mathrm{~mm}$ e altura de $15 \mathrm{~mm}$, até o limite onde o processo de mistura mostrou-se ineficiente.

O processo de fabricação implementado foi o hand lay-up associado com compressão de 2,7 Ton, por um período de 20 min. Foi utilizado molde metálico em aço inox, nas dimensões de 320 x $170 \mathrm{~mm}$. O processo consistiu inicialmente na remoção da umidade superficial da carga mineral em estufa à $105{ }^{\circ} \mathrm{C}$, por 30 min., seguido de sua inserção na matriz com acelerador de cobalto e posterior inserção do iniciador de reticulação. A agitação mecânica foi conduzida por um período próximo ao ponto de gel, 7 min., minimizando, assim, o tempo de inércia do material e a tendência à sedimentação da carga. Após o término do período de compressão, o material foi acondicionado sem tratamento posterior por $24 \mathrm{~h}$ para a finalização do processo de cura e posterior obtenção dos corpos de prova conforme a norma ASTM D 635-03 [14], através da utilização de serra rotativa de bancada.

\subsection{Ensaios de Flamabilidade Horizontal}

A determinação da taxa de flamabilidade dos compósitos foi realizada através da exposição dos corpos de prova padronizados, nas dimensões de 128 x 12,8 x 3,2 mm, à chama controlada. O procedimento consistiu no acompanhamento do tempo necessário para a propagação da chama ao longo dos $75 \mathrm{~mm}$ de área útil, sendo realizadas 3 repetições para os corpos de prova com queima total e 10 (dez) repetições para os materiais em que a chama não se sustentar até atingir a marcação final [14].

\section{RESULTADOS E DISCUSSÃO}

\subsection{Espectrometria de Fluorescência de Raios-X}

A composição química do perfil laterítico estudado pode ser observada na Tabela 1, juntamente com as composições de perfis lateríticos em diferentes regiões. O resultado demonstra um elevado percentual de compostos de ferro agrupados em $\mathrm{Fe}_{2} \mathrm{O}_{3}$ e alumínio, agrupado em $\mathrm{Al}_{2} \mathrm{O}_{3}$, conforme óxido mais estável. $\mathrm{O}$ teor de óxido de titânio $\left(\mathrm{TiO}_{2}\right)$, apesar de baixo quando comparado aos elementos principais, mostra-se expressivo ao ser comparado aos valores encontrados na literatura.

Tabela 1: Comparativo entre a composição química de lateritas de diferentes regiões (\%).

\begin{tabular}{ccccc}
\hline & $\begin{array}{c}\text { Vizeu (Pará) } \\
\text { Este Trabalho }\end{array}$ & $\begin{array}{c}\text { Campo dos Goytacazes (RJ) } \\
\text { Vieira et al. [10] }\end{array}$ & $\begin{array}{c}\text { República Centro-Africana } \\
\text { Nzabakurikiza et al. [15] }\end{array}$ & $\begin{array}{c}\text { Gana } \\
\text { Osei et al. [16] }\end{array}$ \\
\hline $\mathrm{Fe}_{2} \mathrm{O}_{3}$ & 61,13 & 39,34 & 17,71 & 42,33 \\
$\mathrm{Al}_{2} \mathrm{O}_{3}$ & 18,63 & 14,91 & 14,99 & 4,94 \\
$\mathrm{SiO}_{2}$ & 0,66 & 36,5 & 54,29 & 21,81 \\
$\mathrm{TiO}_{2}$ & 5,05 & 0,86 & 0,91 & 0,50 \\
$\mathrm{MnO}$ & - & - & 0,02 & 0,84 \\
$\mathrm{FeO}$ & - & - & 0,43 & - \\
$\mathrm{MgO}$ & - & - & 0,22 & 0,68 \\
$\mathrm{CaO}$ & - & - & 0,03 & 0,12 \\
$\mathrm{Na} 2 \mathrm{O}$ & - & - & 0,09 & 0,57 \\
$\mathrm{~K}_{2} \mathrm{O}$ & - & - & 0,6 & 0,43 \\
$\mathrm{P}_{2} \mathrm{O}_{5}$ & - & - & 0,09 & 0,15 \\
$\mathrm{SO}_{3}$ & - & - & - & 0,01 \\
$\mathrm{Cl}$ & - & - & - & 0,15 \\
$\mathrm{P.F}$. & 14,53 & 8,2 & 9,46 & 27,5 \\
\hline
\end{tabular}

A diferença apresentada entre as composições químicas é advinda do processo de formação dos materiais e das rochas parentais. COSTA [9] ao estudar e definir o conceito de laterita, apresenta uma série de características e composições que podem ser apresentadas por esses materiais, dessa forma, os elevados teores de hidróxidos apresentados pelo objeto de estudo podem não ser similares a outras formações. Contudo, a 
presença significativa de compostos de Ferro e Alumínio despontam como uma característica que justifica a realização da pesquisa.

\subsection{Infravermelho por transformada de Fourier}

A fim de conhecer-se a composição estrutural da laterita e investigar a presença de ligações formadas pelo grupo $\mathrm{OH}$, obteve-se o espectro de FTIR deste minério, apresentado na Figura 2. No domínio de 4000-3000 $\mathrm{cm}^{-1}$, há o aparecimento de bandas características de absorção em $3619 \mathrm{~cm}^{-1}, 3523 \mathrm{~cm}^{-1}, 3448 \mathrm{~cm}^{-1}, 3380 \mathrm{~cm}^{-}$ ${ }^{1}$ e $3115 \mathrm{~cm}^{-1}$, o que caracteriza as vibrações de estiramento de grupos $-\mathrm{OH}$, sendo a região em $3448 \mathrm{~cm}^{-1}$ indicativa da presença de cátions trivalentes de $\mathrm{Al}^{3+}$ ou $\mathrm{Fe}^{3+}$ [17-19], o que é característico dos minerais Gibbsita [20] e Goethita [21]. Os espectros ainda apresentam no domínio de 1750-900 $\mathrm{cm}^{-1}$ bandas em 1019 $\mathrm{cm}^{-1}, 964 \mathrm{~cm}^{-1}, 908 \mathrm{~cm}^{-1}$ que são originárias de vibrações de estiramento Si-O, e uma menos intensa em 1631 $\mathrm{cm}^{-1}$, que pode ser atribuída a moléculas de água presentes em aluminossilicatos [17,22]. Finalmente a região compreendida entre $900-400 \mathrm{~cm}^{-1}$ indica a presença de bandas de absorção em $803 \mathrm{~cm}^{-1}, 735 \mathrm{~cm}^{-1}$ sinalizando a ocorrência de cátions trivalentes, e em $550 \mathrm{~cm}^{-1}$ e $451 \mathrm{~cm}^{-1}$ que se devem as vibrações de estiramento características da presença de ligações Fe-O [19, 22].

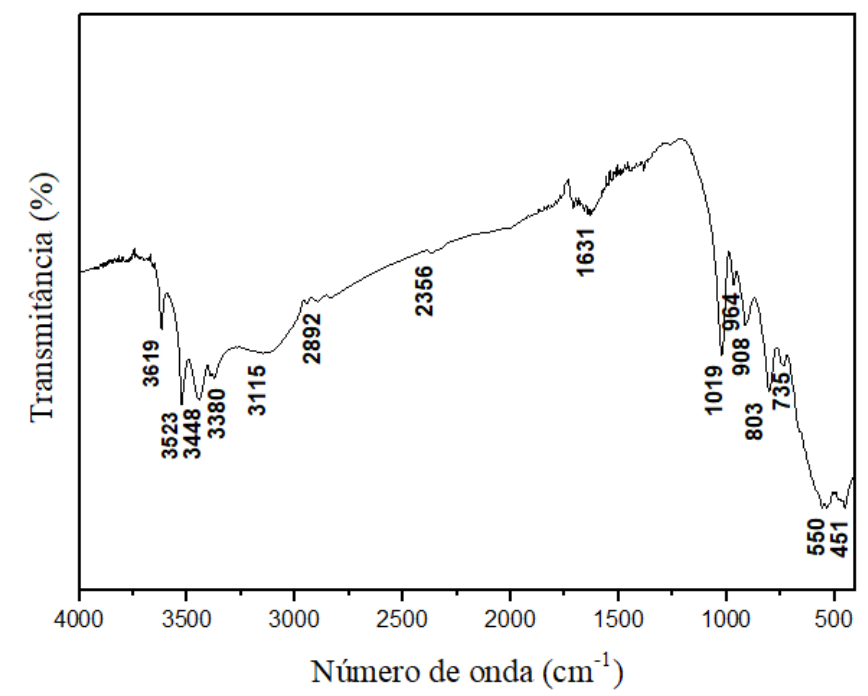

Figura 2: Espectro de Infravermelho para o material laterítico estudado.

\subsection{Granulometria a Laser}

Os resultados obtidos através da granulometria a laser foram expressos em forma do gráfico de frequências e dos diâmetros de referência $\mathrm{D}_{10}, \mathrm{D}_{50}$ e $\mathrm{D}_{90}$, além do diâmetro médio $\left(\mathrm{D}_{\mathrm{M}}\right)$, conforme representado na Tabela 2.

Tabela 2: Diâmetros de referência $(\mu \mathrm{m})$.

\begin{tabular}{cccc}
\hline $\mathbf{D}_{10}$ & $\mathbf{D}_{50}$ & $\mathbf{D}_{90}$ & $\mathbf{D}_{\mathbf{M}}$ \\
\hline 0,6 & 8,6 & 34,4 & 23,5 \\
\hline
\end{tabular}

Analisando os valores dos diâmetros de referência para o material inorgânico nota-se que 90 \% deste material contém partículas com tamanho inferior a $34,4 \mu \mathrm{m}$, o que garante uma faixa de trabalho com diâmetro médio de $23,5 \mu \mathrm{m}$, dentro da região esperada para melhor efeito na flamabilidade, conforme descrito por ÇOBAN [23]. Tais características são de fundamental importância, pois influenciarão em parâmetros como a permeabilidade, resistência e densidade do material, bem como em sua dispersão na matriz polimérica. A Figura 3 apresenta o gráfico com a distribuição granulométrica. 


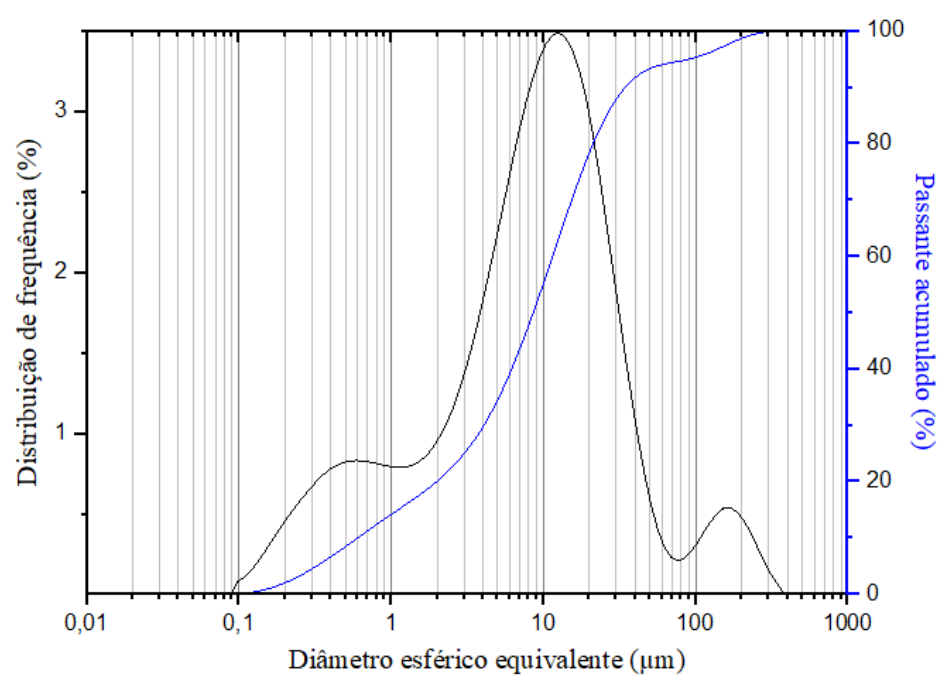

Figura 3: Gráfico de distribuição de granulométrica do material.

Nota-se através da curva de frequência que os materiais apresentam partículas de tamanhos variados compreendidos entre aproximadamente 0,08 e $411 \mu \mathrm{m}$, com modas situadas em 0,47, 12,71 e 168,43 $\mu \mathrm{m}$. De acordo com CHAITREE et al. [24] a redução na granulometria das partículas influência direta e significativamente na transformação de fases sofrida pelos hidróxidos de alumínio e ferro, reduzindo a temperatura de degradação e aumentando a perda de massa resultante, o que será acompanhado através da análise térmica.

\subsection{Análise Termogravimétrica e Calorimetria exploratória diferencial}

Os resultados de TG e DSC foram analisados especificamente dentro da faixa de decomposição do Poliéster Isoftálico, compreendida entre 200 e $550^{\circ} \mathrm{C}[25,26]$. A Figura 4 apresenta o termograma da laterita, variando o fluxo de calor e o percentual de massa em função da temperatura, com a área sombreada delimitando a zona de degradação do polímero apontada pela literatura.

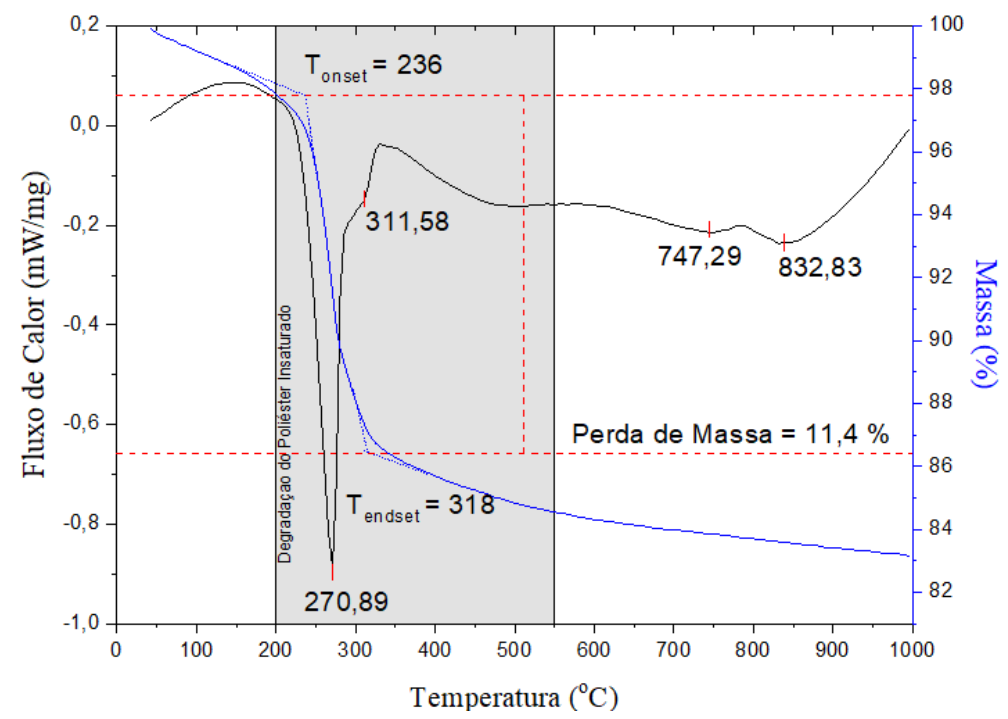

Figura 4: Comportamento térmico apresentado pelo material laterítico com destaque para a região compreendida dentro da faixa de decomposição do Poliéster Isoftálico.

A partir da análise dos resultados foi observado que as principais transformações que resultam em significativa perda de massa ocorrem em uma faixa interessante para o retardo de chamas, coincidindo com a região de degradação do poliéster insaturado. A principal região de perda de massa foi obtida graficamente através da determinação das temperaturas no estágio superior $\left(\mathrm{T}_{\text {onset }}\right)$ e inferior $\left(\mathrm{T}_{\text {endset }}\right)$ da principal região de perda de massa demonstrada na curva de decomposição térmica, correspondendo a mais de $67 \%$ da perda de 
massa total. A Tabela 3 representa as principais informações obtidas pelas análises TG e DSC, dentre elas as temperaturas de perda de massa, temperatura de pico endotérmico e perda de massa total.

Tabela 3: Características do material laterítico para a faixa de decomposição térmica estudada.

\begin{tabular}{ccccc}
\hline $\mathbf{T}_{\text {onset }}\left({ }^{\circ} \mathbf{C}\right)$ & $\mathbf{T}_{\text {endset }}\left({ }^{\circ} \mathbf{C}\right)$ & $\mathbf{T}_{\text {pico }}\left({ }^{\circ} \mathbf{C}\right)$ & $\mathbf{T}_{\text {pico }}\left({ }^{\circ} \mathbf{C}\right)$ & Perda de Massa Total (\%) \\
\hline 236 & 318 & 270,89 & 311,58 & 16,77 \\
\hline
\end{tabular}

O acompanhamento da curva DSC permitiu a obtenção das informações referentes às transformações de fases ocorridas e consequentes perdas de massas. Os picos endotérmicos mais bem acentuados aparecem nas temperaturas de aproximadamente 270 e $311^{\circ} \mathrm{C}$, condizente com as transformações sofridas pela gibbsita e goethita.

A Equação 1 representa a decomposição da gibbsita e consequente formação da alumina de transição $\left(\mathrm{Al}_{2} \mathrm{O}_{3} . k\right)$ com liberação de vapor d'água. A transformação pode ser acompanhada pela significativa perda de massa apontada pelo TG e pelos picos em torno de $270{ }^{\circ} \mathrm{C}$ demonstrados pelo DSC [27-29].

$$
2 \mathrm{Al}(\mathrm{OH})_{3} \rightarrow \mathrm{Al}_{2} \mathrm{O}_{3} \cdot \mathrm{k}+2 \mathrm{H}_{2} \mathrm{O}
$$

A Equação 2 representa a decomposição da goethita e consequente formação da hematita $\left(\mathrm{Fe}_{2} \mathrm{O}_{3}\right)$ com liberação de vapor d'água. A transformação é presentada pelos picos menos acentuados na região em torno de $311^{\circ} \mathrm{C}[20,29,31]$.

$$
2 \mathrm{FeOOH} \rightarrow \mathrm{Fe}_{2} \mathrm{O}_{3}+\mathrm{H}_{2} \mathrm{O}
$$

\subsection{Determinação da taxa de queima}

Os resultados dos ensaios preliminares possibilitaram a fabricação dos compósitos até um limite de $30 \%$ em volume, acima do qual o preenchimento do molde mostrou-se prejudicado pelo aumento na viscosidade da mistura polímero/carga, dessa forma foram produzidos compósitos com 10 \% (LAT10), $20 \%$ (LAT20) e 30 $\%$ (LAT30) de carga mineral. Os resultados do ensaio de flamabilidade horizontal estão apresentados na Tabela 4 onde são representadas as taxas de queima para cada fração e o percentual de redução encontrado para cada compósito em relação ao poliéster insaturado (PI).

Tabela 4: Taxas de flamabilidade para as frações estudadas com seus respectivos erros padrões.

\begin{tabular}{ccc}
\hline Fração & Taxa $(\mathbf{m m} / \mathbf{m i n})$ & Redução $(\%)$ \\
\hline PI & $17,91 \pm 0,49$ & - \\
LAT10 & $14,16 \pm 0,48$ & 20,92 \\
LAT20 & $11,39 \pm 0,60$ & 36,39 \\
LAT30 & $5,40 \pm 1,29$ & 69,86 \\
\hline
\end{tabular}

A análise dos resultados indica uma significativa redução na taxa de queima dos compósitos resultantes da adição de laterita em todas as frações de trabalho, atingido um percentual de até aproximadamente $70 \%$, o que indica um efeito significativo da adição da carga particulada para a propriedade em análise. Conforme os resultados das análises térmicas e da fluorescência de raios-X, esse resultado pode ser associado à composição do material e seus elevados teores de hidróxidos de ferro e alumínio, que sofrem decomposição endotérmica dentro da faixa de decomposição do poliéster, e a liberação de vapor d'água resultante da desidroxilação dos componentes, especificamente gibbsita e goethita, conforme as Reações 1 e 2 e bandas indicadas pelo FTIR, além da substituição de partes dos componentes inflamáveis característicos do polímero por materiais de baixa ignição presentes no particulado. A Figura 5-a apresenta o corpo de prova de poliéster (PI) com o avanço do tempo, onde fica evidenciada a queima completa, em um tempo de aproximadamente 180 segundos. A Figura 5-b apresenta alguns corpos de prova da fração LAT30 ao término do ensaio, onde é possível perceber a influência da carga adicionada e a extinção da chama antes de atingir a marcação final, o que ratifica o efeito positivo para o retardo de chamas. 
a)

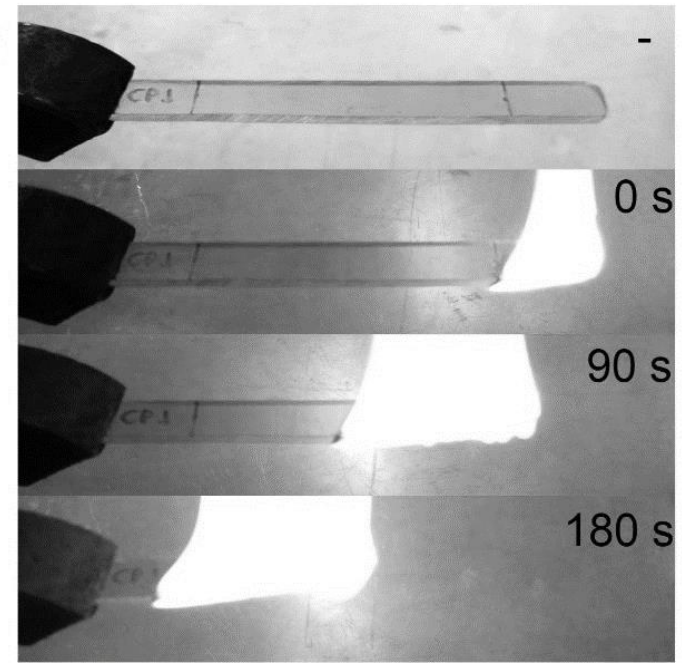

b)

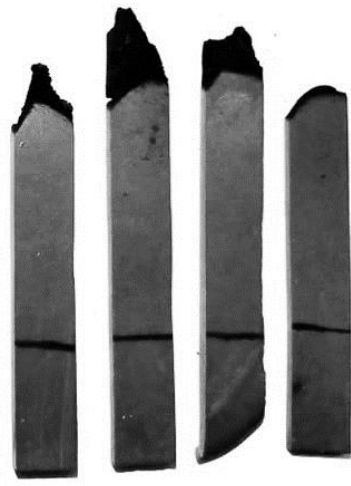

Figura 5: a) Evolução da propagação de chamas com o tempo; b) Corpos de prova da fração LAT30.

De acordo com a Norma ASTM D 635-03 [14], a taxa de queima para materiais com espessura superior à $3 \mathrm{~mm}$ deve ser inferior à $40 \mathrm{~mm} / \mathrm{min}$, estando todas as frações estudadas de acordo com as exigências para a classificação. A Tabela 5 apresenta a comparação entre as taxas do polímero e da melhor fração encontrada no trabalho, com resultados presentes na literatura.

Tabela 5: Comparativo entre as taxas de queima $(\mathrm{mm} / \mathrm{min})$ de diferentes materiais.

\begin{tabular}{lcc}
\hline \multicolumn{1}{c}{ Material } & Taxa $(\mathbf{m m} / \mathbf{m i n})$ & Fonte \\
\hline Poliéster Isoftálico (PI) & $17,91 \pm 0,49$ & Este Trabalho \\
Compósito LAT30 & $5,40 \pm 1,29$ & Este Trabalho \\
Polietileno & $15,20 \pm 0,70$ & ASTM D635-03 [14] \\
Acrílico & $29,70 \pm 1,70$ & ASTM D635-03 [14] \\
Polietileno Tereftalato (PET) & $192,00 \pm 32,20$ & ASTM D635-03 [14] \\
Polietileno /Etileno Propileno Dieno (LPDE/EPDM) & $33,25 \pm 2,35$ & CHANG et al. [32] \\
Compósito LPDE/EPDM/Montmorinolita 60\% & $16,76 \pm 1,48$ & CHANG et al. [32] \\
Compósito LPDE/EPDM/Oxalato de Alumínio 60\% & $10,73 \pm 0,32$ & CHANG et al. [32] \\
Polietileno de alta densidade & $25,31 \pm 0,37$ & BARBOSA et al. [33] \\
\hline
\end{tabular}

O comparativo entre os resultados indica que o polímero utilizado possui uma significativa resistência térmica e a adição da carga inorgânica, em uma fração volumétrica de $30 \%$ (equivalente à $50 \%$ em massa), proporcionou resultados superiores ao demonstrado por CHANG et al. [32] ao utilizar compostos de alumínio e argilominerais. Quanto ao percentual adicionado para a fabricação dos compósitos, fica evidente a redução na taxa com o aumento da fração volumétrica de particulados, conforme representado na Figura 6, onde são representados as taxas de queima e o desvio padrão. 


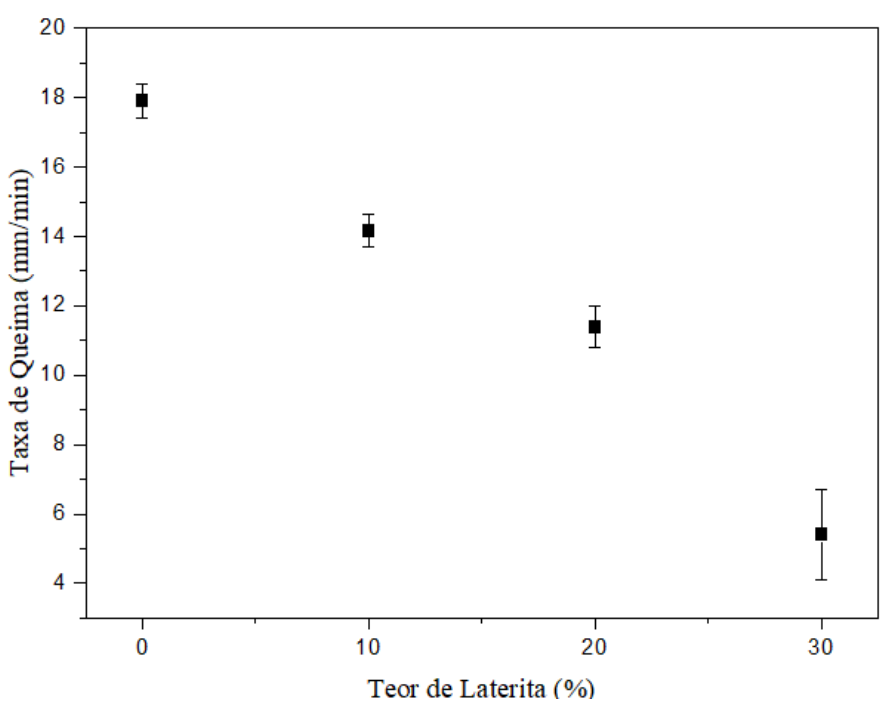

Figura 6: Taxas de flamabilidade em função do percentual de laterita nos compósitos.

\subsection{Tratamento Estatístico - Análise de Variância (ANOVA)}

O tratamento estatístico aplicado foi a análise de variância de fator único (ANOVA) com $95 \%$ de confiança, utilizando a função "Tratamento de dados" disponível no Software Microsoft Excel e no Software Past 3.0, disponibilizado pela Universidade de Oslo. Para a comparação entre as médias foi utilizado o teste de Tukey. A Tabela 6 apresenta os indicadores estatísticos avaliados para a determinação do efeito das variáveis estudadas.

Tabela 6: Indicadores estatísticos para a ANOVA.

\begin{tabular}{lcccccc}
\hline Fonte de variação & SQ & GL & MQ & F & Valor-P & F crítico \\
\hline Entre grupos & 455,58 & 3 & 151,86 & 136,00 & $4,24 \mathrm{E}-11$ & 3,29 \\
Dentro dos grupos & 16,75 & 15 & 1,12 & - & - & - \\
Total & 472,33 & 18 & - & - & - & - \\
\hline
\end{tabular}

De acordo com o resultado demonstrados na tabela anterior, os valores de $\mathrm{F}$ e a probabilidade de significância (Valor-P) indicam que a variação do percentual é significativa, dessa forma, ao menos uma das frações é em média diferente das demais. Para determinar quais frações são estatisticamente diferentes, com um grau de confiança de $95 \%$, foi realizado o teste de Tukey conforme disposto na Tabela 7.

Tabela 7: Comparativo entre as médias (teste de Tukey) com as respectivas diferenças mínimas significativas (DMS) para cada par analisado.

\begin{tabular}{ccc}
\hline Par & DMS & $\left|\overline{\mathbf{y}}_{\mathrm{i}}-\overline{\mathbf{y}}_{\mathrm{j}}\right|$ \\
\hline a-b & 2,4891 & 3,7470 \\
a-c & 2,4891 & 6,5177 \\
a-d & 2,0068 & 12,5114 \\
b-c & 2,4891 & 2,7707 \\
b-d & 2,0068 & 8,7644 \\
c-d & 2,0068 & 5,9937 \\
\hline
\end{tabular}

Para todos os pares analisados a diferença entre as médias é superior à diferença mínima significativa (DMS), dessa forma todas as frações analisadas são diferentes entre si, reforçando o que foi descrito anteriormente.

A Figura 7 apresenta a distribuição dos resíduos em torno do valor normal esperado, a partir da qual pode-se analisar a pressuposição de normalidade e inferir sobre a independência dos resíduos. Através do 
valor da probabilidade de significância - p(normal), pode-se aceitar a hipótese nula (distribuição normal dos resíduos), não havendo razão para duvidar da normalidade dos resíduos.

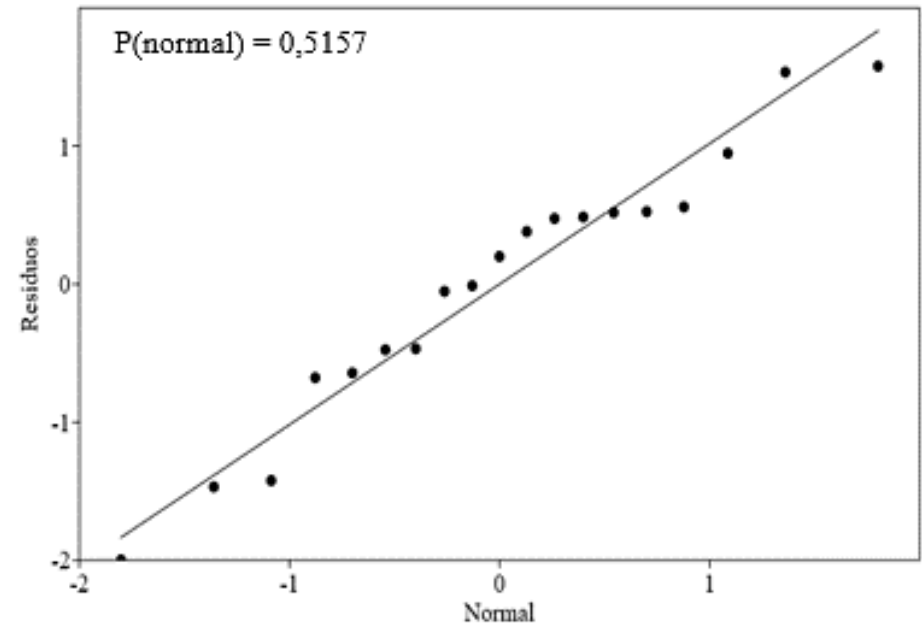

Figura 7: Gráfico de distribuição normal dos resíduos.

\section{CONCLUSÕES}

O material laterítico estudado apresentou um elevado teor de hidróxidos de ferro e alumínio com faixas de decomposição que podem ser associadas às melhorias encontradas. A incorporação de laterita em matriz de poliéster isoftálico foi possível de ser executada dentro de uma faixa pré-determinada de concentração de particulado, garantindo uma boa dispersão e preenchimento do molde metálico.

A fabricação de compósitos com a incorporação de laterita mostrou-se promissora quanto ao retardo de chamas. A redução encontrada nas taxas de propagação com o acréscimo do teor de laterita foi significativa para todas as frações, atendendo ao exigido para a sua classificação HB. A adição do material particulado, na fração máxima adotada, causou um impacto muito positivo e possibilitou a extinção das chamas durante a realização dos ensaios, o que reforça a efetividade do material para a propriedade em análise, embora sejam necessários estudos adequados para a determinação de propriedades complementares de acordo com as possíveis aplicações.

Do ponto de vista econômico e ambiental, foi possível reduzir a quantidade de resina polimérica em um percentual significativo e a adição dos óxidos e hidróxidos presentes no material mineral, em substituição aos compostos já consolidados como retardantes ou auxiliares, reduz a emissão de componentes nocivos associado ao uso de compostos halogenados.

Destaca-se, por fim, que os resultados encontrados durante a realização do estudo não garantem um comportamento similar para todos os materiais lateríticos. É importante destacar que os resultados são creditados principalmente à composição investigada através das técnicas de caracterização aplicadas, dessa forma, dependendo das regiões e composições dos solos, diferentes respostas podem ser obtidas quanto às propriedades analisadas.

\section{AGRADECIMENTOS}

Os autores agradecem ao LaMIGA/IG/UFPA, ao LCM/IG/UFPA, ao LAMPS/FEQ/UFPA, à USIMAT/FEQ/UFPA, à Universidade Federal do Pará, ao CNPq e à CAPES.

\section{BIBLIOGRAFIA}

[1] DAS, A., BAR, M., ALAGIRUSAMY, R., "Flame retardant polymer composites", Fibers and Polymers, v. 16, n. 4, pp. 705-717, 2015.

[2] AL-TAEE, H., FARHAN, A.A., GHULAM, S.H., "Effect of flame retardant additive on the flammability of unsaturated polyester resin. AL-Taqani, v. 26, n. 2, pp. 1-8, 2013. 
[3] TANG, W., ZHANG, S., SUN, J., et al., "Flame retardancy and thermal stability of polypropylene composite containing ammonium sulfamate intercalated kaolinite", Ind. Chem. Res., v. 55, pp. 7669-7678, 2016.

[4] ZHANG, S., JIANG, P., LIU, X., et al., "Effects of kaolin on the termal stability and flame retardancy of polypropylene composite.” Polym. Adv. Technol., v. 25, pp. 912-919, 2014.

[5] FENG, C., ZHANG, Y., LIU, S., et al., "Synergistic effect of $\mathrm{La}_{2} \mathrm{O}_{3}$ on the flame retardant properties and the degradation mechanism of a novel PP/IFR system", Polymer Degradation and Stability, v. 97, pp. 707$717,2012$.

[6] SU, X., YI, Y., TAO, J., et al., "Synergistic effect of zinc hydroxystannate with intumescente flameretardant on fire retardancy and termal behavior of polypropylene", Polymer Degradation and Stability, v. 97, pp. 2128-2135, 2012.

[7] WU, N., DING, C., YANG, R., "Effects of zinc and nickel salts in intumescente flame-retardant polypropylene", Polymer Degradation and Stability, v. 95, pp. 2589-2595, 2010.

[8] LAOUTID, F., BONNAUD, L., ALEXANDRE, M., et al., "New prospects in flame retardant polymer materials: From fundamentals to nanocomposites”, Materials Science and Engineering, v. 63, pp. 100-125, 2009.

[9] COSTA, M.L., “Aspectos geológicos dos lateritos da Amazônia,” Revista Brasileira de Geociências v. 21, pp. 146-160, 1991.

[10] VIEIRA, C.M.F., INTORNE, S.C., ALEXANDRE, J., et al., "Efeito da utilização de laterita nas propriedades físicas e mecânicas de cerâmicas vermelhas”, Revista Matéria, v. 12, n. 3, pp. 446-452, 2007.

[11] QUAST, K., CONNOR, J.N., SKINNER, W., et al., "Preconcentration strategies in the processing of nickel laterite ores part 2: Laboratory experiments." Minerals Engineering, v. 79, pp. 269-278, 2015. ISSN 0892-6875.

[12] CASTRO, R.T., HORBE, A.M.C., ALMEIDA, C.M., “A crosta laterítica ferro-titanífera da Vila Nova Colina e a lateritização no sul de Roraima.” Acta Amaz., v. 46, n. 1, pp. 47-60, 2016.

[13] ZHOU, S., WEI, Y., LI, B., et al., "Mineralogical characterization and design of a treatment process for Yunnan nickel laterite ore, China" International Journal of Mineral Processing, v. 159, pp. 51-59, 2017. ISSN 0301-7516.

[14] AMERICAN SOCIETY FOR TESTING AND MATERIALS, ASTM D 635 Standard Test Method for Rate of Burning and/or Extend and Time of Burning of Plastics in a Horizontal Position, West Conshohocken, 2003.

[15] NZABAKURIKIZA, A., ONANA, V.L., ZE, A.N., et al., "Geological, geotechnical, and mechanical characterization of lateritic gravels from Eastern Cameroon for road construction purposes”, Bull Eng Geol Environ, v. 76, pp. 1549-1562, 2017. https://doi.org/10.1007/s10064-016-0979-y.

[16] OSEI, J., GAWU, S.K., SCHÄFER, A.I., et al., "Impact of laterite characteristics on fluoride removal from water", J. Chem. Technol. Biotechnol., v. 91 pp. 911-920, 2016. DOI:10.1002/jctb.4656.

[17] MELLINI, M., FUCHS, Y., VITI, C., et al., "Insights into the antigorite structure from Mössbauer and FTIR Spectroscopies”, Eur. J. Mineral, v. 14, pp. 97-104, 2002.

[18] SAHU, R.C., PATEL, R., RAY, B.C., "Utilization of activated CO2-neutralized red mud for removal of arsenate from aqueous solutions", Journal of Hazardous Materials, v. 179, pp. 1007-1013, 2010.

[19] JANKOVIĆ, B., SMIČIKLAS, I., STAJIĆ-TROŠIĆ, J., et al., "Thermal characterization and kinetic analysis of non-isothermal decomposition process of Bauxite red mud. Estimation of density distribution function of the apparent activation energy", International Journal of Mineral Processing, v. 123, pp.46-59, 2013.

[20] LEE, D.H., CONDRATE, R.A., "An FTIR spectral investigation of the structural species found on alumina surfaces", Materials Letters, v. 23, pp. 241-246, 1995.

[21] SALAMA, W., EL AREF, M., GAUPP, R., "Spectroscopic characterization of iron ores formed in different geological environments using FTIR, XPS, Mössbauer spectroscopy and thermoanalyses", Spectrochimica Acta Part A: Molecular and Biomolecular Spectroscopy. v. 136, pp. 1816-1826, 2015.

[22] LIU, K., CHEN, Q., HU, H., et al., "Characterization and leaching behaviour of lizardite in Yuanjiang laterite ore", Applied Clay Science, v. 47, pp. 311-316, 2010.

[23] ÇOBAN, O., BORA, M.O., SINMAZÇELIK, T.., "Effect of Mixed Size Particles Reinforced on the Thermal and Dynamic Mechanical Properties of $\mathrm{Al}_{2} \mathrm{O}_{3}$ /PPS Composites", Polymer Composites., v. 37, pp. 3219-3227, 2016. 
[24] CHAITREE, W., JIEMSIRILERS, S., MEKASUWANDUMRONG, O., et al., "Effect of Milling on the Formation of Nanocrystalline $\mathrm{x}-\mathrm{Al}_{2} \mathrm{O}_{3}$ from Gibbsite", J. Am. Ceram. Soc., v. 93, n. 10, pp. 3377-3383, 2010.

[25] BAI, Z., SONG, L., HU, Y., et al., "Preparation, Flame Retardancy, and Thermal Degradation of Unsaturated Polyester Resin Modified with a Novel Phosphorus Containing Acrylate", Industrial \& Engineering Chemistry Research, v. 52, n. 36, pp. 12855-12864, 2013. DOI: 10.1021/ie401662x.

[26] GHENO, G., GANZERLA, R., BORTOLUZZI, M., et al., "Determination of degradation kinetics of two polyester thermosetting powder coatings using TGA and colorimetric analysis", Progress in Organic Coatings, v.78, pp. 239-243, 2015. https://doi.org/10.1016/j.porgcoat.2014.08.015.

[27] ALVES, M.E., MASCARENHAS, Y.P., VAZ, C.M.P., "Comparação de procedimentos de quantificação de caulinita e gibbsita na fração argila desferrificada de Latossolos", Rev. Bras. Ciênc. Solo, v. 32, n. 2, pp. 569-577, 2008. http://dx.doi.org/10.1590/S0100-06832008000200012.

[28] ATASOY, A., "Reduction of ferric oxides in the red mud by the aluminiothermic process", In: International advanced technologies symposium, pp. 2-3, 6, 2011, Elazig, 2011.

[29] MERCURY, J.M.R., GALDINO, L.G., VASCONCELOS, N.S.L.S., et al., "Estudo do comportamento térmico e propriedades físico-mecânicas da lama vermelha", Matéria (Rio J.), v. 15, n. 3, pp. 445-460, 2010. http://dx.doi.org/10.1590/S1517-70762010000300007.

[30] CARNEIRO, B.S., ANGÉLICA, R.S., SCHELLER, T., et al., "Caracterização mineralógica e geoquímica e estudo das transformações de fase do caulim duro da região do Rio Capim, Pará." Cerâmica, v. 49, pp. 237-244, 2003.

[31] MAGALHÃES, F., Síntese e caracterização de óxidos de ferro e compósitos para aplicações no tratamento redox de efluentes aquosos, Tese de D.Sc., UFMG, Belo Horizonte, MG, Brasil, 2008.

[32] CHANG, Z., GUO, F., CHEN, J., et al., "Synergic flame retardancy mechanism of montmorillonite in the nano-sized hydroxyl aluminum oxalate/LDPE/EPDM system", Polymer, v. 48, n. 10, pp. 2892-2900, 2007. https://doi.org/10.1016/j.polymer.2007.03.044.

[33] BARBOSA, R., ALVES, T.S., ARAUJO, E., et al., "Flammability and morphology of HDPE/clay nanocomposites", Journal of Thermal Analysis and Calorimetry, v. 115, pp. 627-634, 2013. DOI: 115. 10.1007/s10973-013-3310-1.

\section{ORCID}

Bruno Henrique Alves Mendes

Sylvester Stallone Pontes Sales

Lesley Glenda Sales dos Santos

Edinaldo José de Sousa Cunha

João Nazareno Nonato Quaresma

José Antônio da Silva Souza https://orcid.org/0000-0002-3770-0364

https://orcid.org/0000-0003-2128-3129

https://orcid.org/0000-0002-1473-5847

https://orcid.org/0000-0001-8047-6786

https://orcid.org/0000-0001-9365-7498

https://orcid.org/0000-0002-1784-9217 